\title{
Electrocardiograma fetal intra-uterino
}

Doctores Rafael Peralta Cayón, profesor de obstetricia; Alva_ ro Velasco, Jefe de Clínica Obstétrica (Facultad Nacional de Mie dicina de Bogotá) y Marcos Duque Gómez, del Departamento de Cardiología del Hospital de San Juan de Dios, de Bogotá.

Trabajo presentado a la III Convención Nacional de Obstetricia y Ginecología reunida en Ibagué, Colombia, del 4 al 7 de diciembre de 1957

Orden.-Generalidades, significación, anoxias, experiencias, resumen y resultados, bibliografía.

\section{GENERALIDADES}

Doble derrotero nos ha guiado en el presente ensayo que tildamos de comunicación inicial y aspiramos a que no quede trun. cada: la constante preocupación por velar y asegurar la vida del feto in_útero, y resaltar un medio de exploración científica que demina la clínica cardiológica.

Siempre nos hemos rebelado contra la indiferencia que pue. da existir ante la supervivencia del feto, como se observa en enti. dades de patología obstétrica, por ejemplo, el caso de las toxe'mias, en que las escuelas que denominamos avanzadas o moder. nas, al sustentar y garantizar la necesidad del tratamiento pura. mente médico, no dejan de subestimar la suerte que coja el hijo, tal como lo atestiguan los balances estadísticos.

Por ello todos los recursos a que apelemos en beneficio del producto de la concepción, no son redundantes, y uno de éstos es el registro electrocardiográfico del corazón fetal a través de la madre, procedimiento al cual hemos llegado con la mira de despejar algunos estados morbosos o no, de la vida intrauterina, después de vencer una serie de dificultades, como quiera que no es un sistema sencillo debido a los conocidos obstáculos que impi. 
den abordar la actividad eléctrica del miocardio del feto, estan. do él dentro del útero, rodeado de tantas interferencias y de ele. mentos que tratan de aislarlo, más la lectura e interpretación del electrocardiograma correspondiente, que pueden ser más o me_ nos complejas.

El origen del aparato circulatorio es mesodérmico; en el pe_ ríodo genético de huevo, desde $\operatorname{los} 15$ a 40 días de la fecundación se aprecia un punto que late y equivale al futuro corazón (que primitivamente es doble) con canales arteriales arriba y venas onfalomesentéricas abajo, que más los canales vertebrales, constituyen los tipos de circulaciones vitelina y alantoidea del huevo. embrión. A los 40 días se establece la circulación placentaria con cuyo perfeccionamiento se completa la formación anatomo_histo_ lógica y fisiológica del miocardio, hasta organizar los sistemas de circulaciones mayor y menor que se consolidan después del nacimiento, pero entre el tercero y cuarto meses lunares ya se obser. va el corazón adherido a la futura cavidad torácica, al quinto mes lunar se esbozan ruidos cardíacos embriocárdicos y taquicárdicos a la apreciación clínica, y desde la vida embrionaria existe un auténtico complejo electrofisiológico del corazón, tal como se ha ratificado por medio de derivaciones para electrocardiogramas en fetos de $91 / 2$ a 25 semanas, y hasta en embriones apenas de 6 milimetros, al notarse deflexiones concordantes con el complejo QRS y onda T, sabiendo, claro está, que a mayor edad del feto dicho registro será mejor, razón por la cual Easby en 1934 hubie_ ra practicado un electrocardiograma en un feto de $4 \frac{1}{2}$ meses, pa. ra que también Raiha en 45 prematuros de 1.000 a 2.500 gramos de peso, comprobara la coincidencia con el complejo electrocardio. gráfico embrionario, y las observaciones de Vara y Niemineva en el curso de cesáreas, fijaran QRS de 0,02 a 0,03", e intervalo PQ ce 0,05 a 0,07 , a todo lo cual se agrega la experimentación en embriones de pollo, en los cuales se han obtenido QRS definidos y $\mathbf{T}$ pequeñas.

En el recién nacido los electrocardiogramas no ofrecen pro_ blema mayor en cuanto a técnica se refiere, y Alberto Vejarano Laverde, en 100 observaciones comprobó en general los resulta_ dos del niño normal: ritmo sinusal taquicárdico entre 100 y 113 , $T$ negativa o isoeléctrica en derivaciones I o II en el $42,5 \%$, que podría ser propio de una alteración miocárdica, fue exclusivo al día del nacimiento o con un dia de diferencia, pero que no se atri. buye a lesión patológica legitima; longitud de la onda $\mathbf{P}$, duración 
de 0,04 segundos en las 3 derivaciones; intervalo PR, tiempo de conducción aurículo_ventricular 0,09 a 0.10 segundos en las 3 de_ rivaciones; en cuanto al complejo ventricular, onda $Q$, duración de 0 en derivación I, de 0,0 I en II y III derivaciones, con ampli_ tud de 0 en I, menos 0, I a 0 ,I en II y 1,5 a 0,2 milímetros en III, sa. biendo que $\mathrm{Q}$ no es constante comprobarla, pero su presencia es normal en II y III; longitud de onda $\mathbf{R}$, prominente en todas las derivaciones, sobre todo en III, su aplanamiento se halla en el tercio de los corazones normales, sus escotaduras son de frecuen. cia menor, duración de 0,01 segundos, 0,02 y 0,03 segundos en I, II y III, respectivamente, con las amplitudes correspondientes de menos 0,1 a $0,1,0,1$ a 0,3 y 0,4 a 0,7 milímetros; longitud de onda S, $0.03,0,02$ y 0,00 en I, II y III, con amplitud de 04 a 06 mili. voltios en I, enorme dispersión en II y 0 en III, al tiempo que se recuerda que dicha onda no es constante y cuando existe tien. de a atenuarse con la edad; espacio o complejo QRS, tiempo de conducción intraventricular de 0,04 a 0:05, sabiendo que después del nacimiento y de los primeros años de vida, la desviación ma. yor del grupo QRS es negativa en I, y que la duración promedio de él es de 0,06, espacio $\mathbf{S T}$, isoeléctrico y positivo ascendente, mi. de menos de un milimetro, puede tener ligeras desviaciones ascen. dentes o descendentes; longitud de onda $\mathbf{T}$, suele dirigirse hacia arriba en las 3 derivaciones, en la III se puede admitir sin inver. sión, ya que se encuentra en el tercio de los niños normales, y además se cree que a veces presenta muescas, duración de 0,06 amplitud máxima en II y mínima en III; en cuanto al espacio QT, se ha fijado en 0,25 a 0,32 segundos, y el eje eléctrico normal equivale a +104 a 133 grados en el $46 \%$.

Respecto al electrocardiograma normal del niño, Julio Sán_ chez Acuña, (sobre 10 experimentaciones con 6 puntos precordia. les), asevera que la amplitud de variaciones normalies en el ca. rácter de la imagen electrocardiográfica es muy dilatada, sin que se puedan determinar sus límites, y destaca las observaciones del profesor Luis Forero Nougués, cuyas conclusiones con las extran. jeras se sintetizan asi: ritmo sinusal taquicárdico entre 100 y 110; eje eléctrico, desviado a la derecha constantemente al nacer y en lactantes muy pequeños; onda $\mathbf{P}$, el complejo auricular se mani. fiesta por una deflexión positiva en todas las derivaciones, puede estar invertida en III derivación en el $10 \%$ de los niños nor. males, en general tiene un contorno liso redondeado pero puede ofrecer muescas en alguna derivación en trazados normales, su longitud varía de 0,04 a 0,08 y su altura no pasa de 1 milímetro; 
intervalo $\mathbf{P R}$, el tiempo de conducción aurículo_ventricular se ha. lla entre 0,08 y $0,14{ }^{*}$ para los colombianos y 0,16 a $0,18{ }^{*}$ para los extranjeros; complejo ventricular, onda $Q$, su presencia es normal en II y III derivaciones, longitud de 0,01" y amplitud de 0,1 a 0,2 milivoltios, onda $\mathrm{R}$ de dimensiones que dependen de las des. viación del eje eléctrico a la derecha, con altura máxima en III derivación entre nosotros y en II para los extranjeros, onda $\mathbf{S}$ que no es frecuente y va desapareciendo con la edad en I deriva. ción; espacio QRS, el tiempo de conducción intraventricular es de 0,04 a $0,08: ;$; segmento ST, isoeléctrico, con ligeras desviaciones descendientes o ascendentes y mide menos de 1 milímetro; onda $\mathbf{T}$, va hacia arriba en todas las derivaciones, en III en casos norma. les está invertida más o menos en el tercio de los niños y su altu_ ra no pasa de 1,5 milímetros; espacio QT, mide entre 0,18 y 0,32 . para una frecuencia cardíaca de 120 .

\section{SIGNIFICACION PATOLOGICA: ANOXIAS}

No se escapa la trascendencia de un electrocardiograma fetal previo en variados estados morbosos, especialmente para determi. nar de modo precoz la anoxia o afixia intrauterina, o sufrimien. to fetal, entidad que a pesar de los propios signos clínicos que la identifican, aún traduce un problema tal vez por carencia de oportunidad diagnóstica, y entonces el refuerzo que le proporcio. ne el laboratorio experimental constituye valiosa aportación, ya que para lo que antecede, las estadísticas norteamericanas le asignan el $37 \%$ de toda la mortalidad peri_natal, y por ejemplo sobre 11.147 muertes intrauterinas $92,8 \%$ fueron antes del nacimien. to en contra de $1,4 \%$ defunciones por anoxia después del parto.

La anoxia es un estado de oxidación insuficiente o nulo, más hipercapnia, con grave repercusión sobre los aparatos circulato_ rio, respiratorio y nervioso. Habitualmente se manifiesta por bra. di o taquicardias, arritmia, soplo, más expulsión de meconio por peristaltismo intestinal nervioso o reflejo, y diesde un punto de reparo etiopatogénico, es sabido que el requerimiento de oxígeno progresa con el desarrollo fetal, razón por la cual al término existen $4 \frac{1}{2}$ milímetros por minuto y kilo de peso, al tiempo que su difusión se facilita porque al declinar la placenta el espesor del sincicio desaparece, además de que aumentan los eritrocitos y la hemoglobina. Mas se pueden agregar estados patológicos que criginan algunos tipos de anoxia, como los siguientes: anoxia anóxiça o anoxémica, cuando disminuye el oxígeno circulante ma. terno debido a afecciones circulatorias con insuficiencia cardíaca, 
respiratorias agudas o crónicas, anemias intrínsecas al embarazo o por metrorragias gestantes como inserción baja de la placenta, desprendimiento de ésta normalmente insertada, toxicosis ané. mica, hipotensión o retardo circulatorio de la anestesia raquídea; anoxia materno-fetales, por rémora o estancamiento, cuando no llega al feto el oxígeno aunque exista en la madre, o bien no sale de ella, en los infartos placentarios, circulares o precedencias del cordón umbilical; anoxias anémicas, de los trastornos sanguíneos fetales que impiden el aprovechamiento die oxígeno, como en las enfermedades hemolíticas y eritroblastosis, $\mathrm{Rh}$, etc.; a noxias his_ totóxicas, en las cuales no se absorbe el oxígeno por lesiones his_ toquímicas celulares, producidas por la acción de analgésicos, anestésicos, espamolíticos, tóxicos, envenenamientos, etc.

Los atrayentes estudios de Edward M. Southem (Departa. mento de Obstetricia y Ginecología del Hospital Monte Sinaí de Nueva York), han relacionado la anoxia fetal con las modifica. ciones electrocardiográficas que se aprecian in_útero durante el embarazo y trabajo, con observaciones en la hipermadurez fetal lo mismo que en las toxemias agudas y crónicas, más el comple. mento después del parto que dan los respectivos análisis de sa_ turación de oxígeno en la sangre de los vasos umbilicales por el método de Van Slyke. La técnica consistió en emplear un sistema vector (Samborn 182) con dos canales amplificadores provistos para registros fono y electrocardiográficos fetales, separados o si_ multáneos, amplificación equivalente a 25 milímetros por mili. voltio, mas un filtro especial para bajas frecuencias sobre mínimas ondas respiratorias; un sistema selector y un osciloscopio (Samborn 169) que facilita la apreciación visual selectiva, y en. tre el cual y el vector se destacan los desplazamientos y "regis. tros unidades". Los electrodos se aplican sobre el abdomen ma. terno, dibujan un triángulo cuyo vértice superior queda arriba del ombligo, y los dos inferiores en relación con las espinas ilía_ cas anterosuperiores derecha e izquierda, para así englobar fácil y topográficamente el corazón fetal, por ejiemplo, en una pre. sentación cefálica, vértice izquierdo, y luego se controlan las co. nexiones y derivaciones conocidas, para verificar los registros electrocardiográficos.

En 96 observaciones publicadas, 4 grupos son fundamentales: primero, 46 casos de embarazo y trabajos normales, complejo fe. tal rápido, frecuencia 127, $\mathbf{P}$ amplitud 1/2 milímetros, $P R$ dura. ción 0,06 segundos, QRS con deflexiones, amplitud 8 a 10 milime. tros, duración 0,02" a 0,04", ST duración de 0,09, T cuando se dis_ 
tinguió tuvo amplitud de 0,03 ; los fetos nacieron vivos, sin anoxia, bajo anestesia ligera inhalada, y 30 gasometrías en sangre arte. rial del cordón, a los 2, 4, 8 y 10 minutos del parto, demostraron que las variaciones de saturación de oxígeno fueron a los 2 minu. tos $47,5 \mathrm{v}+0-5,1 \%$ y a los 10 minutos $78,5 \mathrm{v}+0-2,1 \%$. Segundo, 22 casos anormales por trastornos intrauterinos, de rutinaria apre. ciación clínica, con frecuencia de 102, P invertida y de amplitud 1,1 milímetros, PR alargado a 0,12", QRS de voltaje disminuído y duración 0,06 ", ST isoeléctrico o deformado en el $75 \%$ y con duración de 0,12 ", ondas $\mathrm{T}$ bajas o invertidas en derivaciones $\mathrm{I}$ y II en un $35 \%$, de todo lo cual se deduce que en las anoxias los cambios notorios fueron en ST y T, de manera progresiva, pro. longación isoeléctrica e invertida y depresión de ST en circuns. tancias de anoxia grave, sabiendo que tales modificaciones son anteriores al diagnóstico clínico de la bradicardia y que no siem. pre ceden al suministro de $100 \%$ de oxígeno a la madre, para lue_ go concluir que la saturación de oxígeno arterial en 17 anomalías electrocardiográficas previas, fue de $\mathbf{1 \% . 1 v}$ más o menos $4,1 \%$ a 10 s 2 minutos y $\mathbf{4 0 , 5}$ s más o menos $1,9 \%$ a los 10 minutos. Tercero, 20 toxemias, 12 agudas y 8 crónicas. No se registraron variaciones en QRS, PR ni ST, ni anoxia progresiva agravante que obligara por lo tanto a desocupar el útero. Cuarto, 8 historias de hiperma. durez fetal (calculada por edad correspondiente a 295 días des. pués de la última regla), frecuencia $118, \mathrm{P}$ amplitud 0,7 milíme. tros en cierta relación con el ritmo descrito, PR varió de 0,14 " que fue el mayor, en 4 casos, a 0.09 que fue el menor para los res_ tantes, QRS en derivación I tuvo 0,05" de duración, ST deprimi. do en 3 observaciones, $T$ pequeña o isoeléctrica, también en 3 ca_ sos, para que se comprobara así la certeza de anoxia por el elec. trocardiograma fetal y se ratificara con la saturación de oxígeno a los 2 minutos del nacimiento, que demostró un promedio $\%$ de $30,1 \mathrm{v}$, más o menos 3,1 .

\section{NUESTRAS EXPERIENCIAS Y SUS TECNICAS}

Las limitadas observaciones que reunimos pertenecen, menos una, a embarazadas del Instituto de Protección Materno. Infantil de Bogotá. La primera experiencia se cumplió durante más de 1 hora, el 8 de julio de 1957 en dicho Instituto, y las si. guientes en el Laboratorio de Fisiología de la Facultad Nacional de Medicina; se trataba de E. V., historia 44379, primegestante casi a término, O.L., auscultación $\left({ }^{+}\right)$; con un electrocardiógrafo 
Sanborn dirigido por uno de nosotros, como Jefe del Departamen. to de Cardiología del Hospital San Juan de Dios, se intentó ha. cer el registró por vía abdominal con la técnica narrada por los americanos, pero con cierto fracaso porque la sola frecuencia del trazado comprobó que el ECG (abreviatura de electrocardiograma) resultaba casi absolutamente materno, debido a insuficien. cia en el aparataje, falta de sistemas amplificadores, aunque se vis_ lumbraron algunos complejitos fetales, sin que se pudiera estu. diar el ECG fetal por falla en amplificación (gráfica, slide, 1).

Después de analizar las causas del resultado negativo ante. rior, y de cambiar ideas sucesivamente, pasamos al Laboratorio de Fisiología, y el 5 de octubre de 1957 durante más de tres horas, en 3 gestantes dirigió la experimentación el doctor Fernando Rosas, profesor Auxiliar y Encargado de Fisiología, desde el Departamento Electrofisiológico del Laboratorio de Neurofisiología. Mediante un Electrocardiógrafo Cambridge, un Osciloscopio Du. mont 333, acoplado al Electroencefalógrafo Grass modelo 3D de 8 amplificadores P_5, hasta obtener las mejores observaciones de los potenciales electrocardiográficos sobre nitidez, definición de potenciales fetales y ausencia de artificios. Así se aplicaron los electrodos abdominales, pequeños como para electroencefalogra. ma, que circunscribieron el triángulo ya nombrado, más los cono_ cidos para miembros inferiores y superiores maternos y el de tie. rra, pero los registros fetales no fueron satisfactorios porque el ECG materno era el que primaba, no obstante de que quedaron dudas sobre interferencias fetales, de acuerdo con las correspon. dientes lecturas realizadas por los doctores Hernando Ordóñez, Rafael Mendoza y por nosotros (Las historias clínicas anteriores pertenecen a N. M., primigestante $81 / 2-9$ meses, O. I. sin enca. jar, Ausc. positiva. M. V. de M., secundigestante de 45 años de edad, con antecedente de un aborto, embarazo de 8 meses, pelvis ausc. (+), y M. R., tercigestante de $81 / 2$ meses, O. I., aus. $\left(^{+}\right)$.

Luego en noviembre 10, durante experimento continuo de más de 2 horas en una primigestante de $81 \frac{1}{2}$ meses, O. I., ausc. $\left(^{+}\right)$, M. J. de Z., con el uso exclusivo del electroencefalógrafo, 4 electrodos pequeños abdominales para el triángulo y tierra, y 2 maternos cubitales, se logró un resultado halagador con registros mixtos, como quiera que en el osciloscopio se notaron las hondas habituales maternas para electrodos cubitales, y progresivamen. te débiles, fetales para los abdominales, lo cual indicó que se ob. tuvo ei primer ECG fetal, asociado al materno. 
Posteriormente, noviembre 14, con 4 electrodos pero de am. pía superficie y gruesos, en 2 observaciones hubo éxito rotundo, puesto que en papel para electroencefalograma se registraron los respectivos ECG fetales, nítidos, independientes, con complejos QRS perfectos, también debido al valioso y electivo recurso del electroencefalógrafo y con los electrodos y derivaciones de la ex. ploración osciloscópica, tal como se confirma en las gráficas 2,2 A y 3 (Las historias son de Isabel Galindo, gráficas 2 y 2. A, primi. gestante casi a término, O. D., ause. $\left({ }^{+}\right)$, con solo 3 electrodos para el triángulo, quien más tarde tuvo el parto con cesárea por esproporción céfalo.pélvica, masculino de 3.200 gramos, respira. ción normal; y Hortensia Vega, gráfica 3, primigestante a térmi. no, en trabajo inicial, O. I. insinuada, ausc. $\left({ }^{+}\right), 4$ electrodos, 3 para el triángulo y 1 para tierra, cuyo parto se hizo el mismo día espentáneamente, masculino de 3.000 gramos, respiración normal).

La interpretación del ESG, nada especial ni anormal demos. tró en estos dos casos, en el primero de los cuales se dedujeron los datos siguientes: frecuencia fetal, 125; materna, 90; P, 1 milí. metro; R, 5 milímetro; $S$, 1i $\frac{1}{2}$ milimetro; T, 1 milímetro; PR, 0,04 "; QRS, 0,04" y QT, 0,12".

En noviembre 21 con la técnica anterior se experimenta en la señora $\mathrm{L}$. de C. (clienta particular), primigestante de 9 meses largos, puesto que la U. R. había sido en febrero 8, la altura ute. rina $34 \frac{1}{2}$ centímetros, O. I., sin encajar. Se logró el ECG fetal y llamó la atención la existencia de un voltaje disminuído, QRS al_ go ancho y deformado; PR, alargado; ST, como deprimido y T, casi negativo, es decir, no parecía tan limpio el registró como las dos observaciones anteriores. El parto terminó normalmente, 4 días después, inducción con pitocín en suero, masculino de 3.350 gramos, talla, 511/2 centímetros; respiración normal.

En seguida, noviembre 22, se practicaron 2 trazados electro. cardiográficos fetales; también con el electroencefalógrafo, pero recurriendo a más de 4 electrodos de amplia superficie que se pu. sieron en diferentes sitios del abdomen y dibujaban diferentes fi. guras al triángulo clásico. El primer caso corresponde a I. B. nú. mero 73913, secundigestante casi a término, O. I. móvil; ausc., $\left.{ }^{+}\right)$; pre_eclampsia, grave; con TA $18 \times 10$ en noviembre 19 , tra. tada médicamente con régimen y largactil, con lo cual mejoró, y la TA bajó a 15 x 10. El ECG resultó algo difícil por posibles contracciones uterinas o movimientos fetales, y los complejos sa_ lian pequeños con voltaje descendido, pero a los 40 minutos de haber iniciado el experimento y después de haber colocado hasta 
7 electrodos que se cambiaban sucesivamente, se logró el registro electrocardiográfico con buenos complejos QRS, frecuencia de 12 latidos en 5"; también PR y QRS como anchos. ST, algo depri. mido, y $\mathrm{T}$, dudosamente negativa con la técnica del triángulo, pe. ro invertido, o sea con el ángulo inferior hacia el pubis y los 2 su. periores hacia los flancos, a todo lo cual se agrega que el parto se nealizó normalmente el 1.o. de diciembre, femenino de 3.100 gramos; respiración, espontánea. Eil 20. caso es el de R. M. A., número 43875, secundigestante de $8.81 / 2$ meses, pelvis, hidrocefa. lia, diagnosticadas con radiografías de octubre 23 y noviembre 19 . El ECG fetal resultó más difícil de lo que pudiera esperarse, tra. tándose de una presentación que no era cefálica; los complejos QRS fueron notorios y ninguna anormalidad cardíaca pudo com. probarse en el feto, más o menos teratológico, aunque QRS y ST estaban algo anchos y deprimidos respectivamente, y $\mathrm{T}$, parecía como difásica, menos más, tal como puede acontecer en isquemias o anoxias miocárdicas sin perder de vista que el triángulo que de dibujó también quedó invertido, y que en diciembre 6 de 1957 aún no se habia verificado el parto a pesar de haber practicado dos inducciones (gráficas 5 y 6 ).

Nota.-Es fundamental saber para cálculos y medidas, que se usaron amplificaciones de 7 milímetros por 5 microvoltios, velo_ cidades del papel de 15,30 y 60 milímetros por segundo, y que pa. ra evitar fluctuaciones de la línea de base se utilizaron en oca. siones constantes de tiempo de electromiografía.

Finalmente, en noviembre 29, quisimos volver al registro con el solo electrocardiógrafo y con un aparato Cambridge dirigido por el doctor Deil Portillo, 60 ciclos, milivoltaje standardts, 1 cen. tímetro equivalente a 1 milivoltio de desplazamiento de la aguja, no se pudo obtener el ECG fetal perfecto, después de haber tra. bajado durante más de 1 hora, con los electrodos de amplia su. perficie para delimitar el triángulo narrado, o el de Einthoven, y por la situación y posición del feto circunscribir los electrodos a la topografía de los miembros superiores e inferiores del feto, como para un registro extra-uterino. Se trataba de A. R. C., número 46826, primigestante de 8.81/2 meses, O. I. ? (grasa abun. dante), ausc., $\left.{ }^{+}\right)$; pre noviembre, que hoy estaba en $15 \times 10,96$ pulsaciones, tratamien. to médico, aunque leyendo más detenidamente el trazado, se ob_ servó grueso, como por calentamiento excesivo de la aguja y quedó la duda de la presencia de uno que otro complejo fetal (grá. fica 7 ). 


\section{RESUMEN Y RESULTADOS}

Se intentaron 11 ECG fetales por medio del electrocardiógra_ fo y electroencefalógrafo, de los cuales 5 fueron perfectos, indu. dables, aislados, y diferentes a los maternos, pero con la utiliza. ción del electroencefalógrafo (gráficas 2, 2-A, 3, 4, 5 y 6). Hubo 6 imperfectos o casi nulos, 2 con elctrocardiógrafos en que primó el registro materno y apenas hubo rudimentos de complejos fe tales indescribibles por falta de amplificación especial, (gráficas 1 y 7), 3 casos con electrocardiógrafo más electroencefalógrafo y osciloscopio, que en éste tradujeron dudas de interferencias feta. les porque primaba también el ECG materno y un caso francamen. te mixto, con electroencefalógrafo, donde hubo fusión con el ma. terno, pero siempre existió el fetal, todo lo cual hace deducir que fue francamente positivo el ECG fetal cuando se empleó el electroencefalógrafo con electrodos de amplia superficie, que dibuja. ron un triángulo relacionado con el ombligo y espinas ilíacas an_ tero_superiores maternas, de vértice mediano y superior, o un trián_ gulo invertido con el vértice inferior hacia el pubis y los dos la_ terales cerca a los flancos denecho e izquierdo.

Los 5 casos positivos francos se distribuyeron asi: 2 obstétri. camente fisiológicos antes del ECG y éste resultó normal en ambos, con un parto mediante cesárea y otro espontáneo, fetos con respiración espontánea; 3 subnormales o anormales, obstétrica. mente considerados, en los cuales los respectivos ECG posteriores parecieron algo irregulares, voltajes descendidos, QRS ancho 0 deformado, PR, prolongado; ST, deprimido; T, como negativa; casos que pertenecieron a una hipermadurez probable con parto espontáneo y feto que respiró normalmente, una toxemia que re. sultó benigna, parto y respiración espontáneos, y una hidrocefa_ lia que aún no ha hecho el parto, sin que se pueda determinar una correlación recíproca desde un punto de vista patológico, ni se pueda prediagnosticar una anoxia, tanto más cuanto que fue imposible la gasometría post_parto, y aún faltan muchas experi. mentaciones para fijar si es cierto que existe una atipicidad sis. temática de ST y $\mathbf{T}$, como lo aseguran los americanos.

Las causas de error en los registros o interferencias fueron escasas, como nerviosismo, contracciones musculares uterinas o extrauterinas, líquido amniótico, movimientos, etc., y en relación a esto la línea de base fue casi siempre perfecta.

Por ahora no podemos ser categóricos en el diagnóstico de la anoxia por el electrocardiograma fetal pre_natal, y ojalá que en 
el futuro se venzan los escollos actuales para complementar el presente estudio que hemos programado para una amplia serie de entidades de patología obstétrica, materna, fetal y ovular, so. bre todo para nuestras toxemias, las más graves entre las que se conocen, y sería interesante el ECG del feto en ellas, a fin de re. solver nuestra perenne inconformidad frente a la probable dis. plicencia que pueda observar el tocólogo ante la salvación del hijo de la pre_eclámptica o eclámptica graves, ya que si se llega. re a descubrir una anoxia irreductible, seguramente el cambio de criterio debería imponerse, pero aquí como en otros estados morbosos, con la esperanza de que el ECG fetal sea un recurso técni. co y científico que no se preste a exagerados intervencionismos.

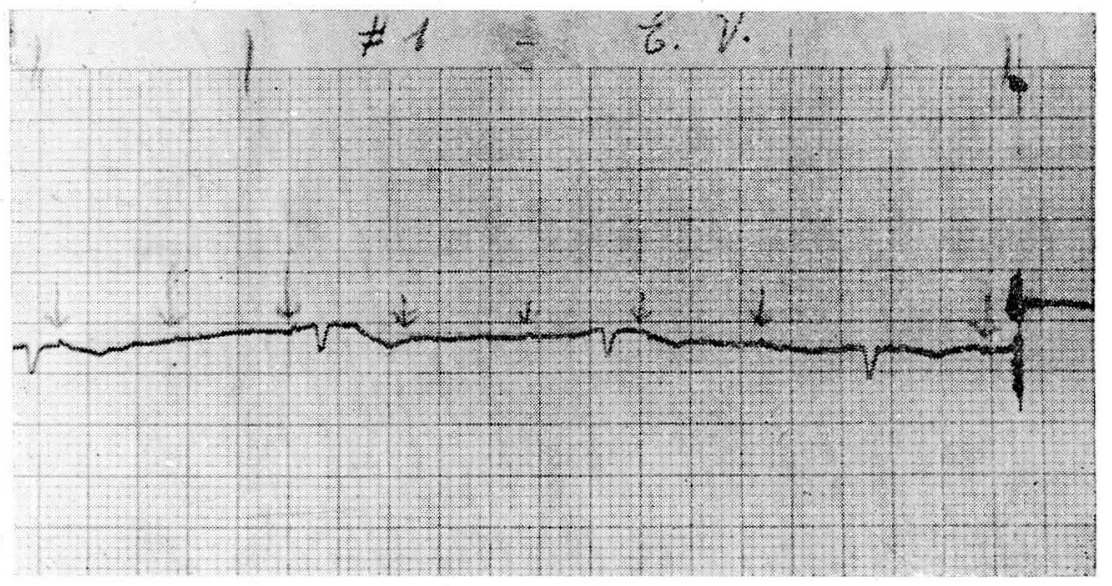

FIGURA NUMERO I

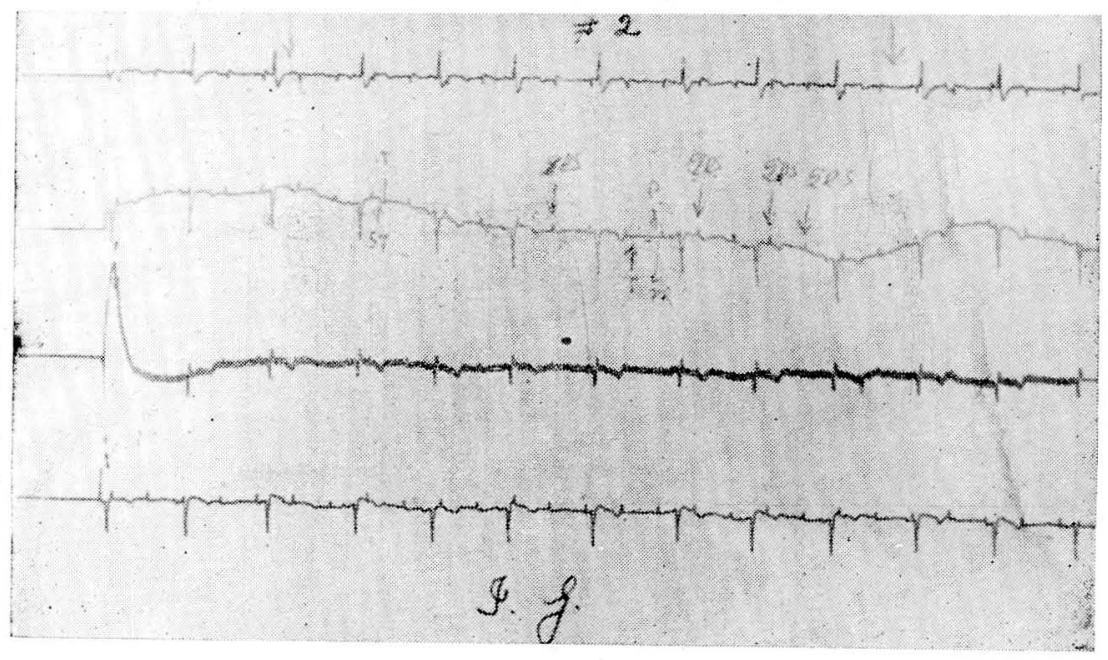




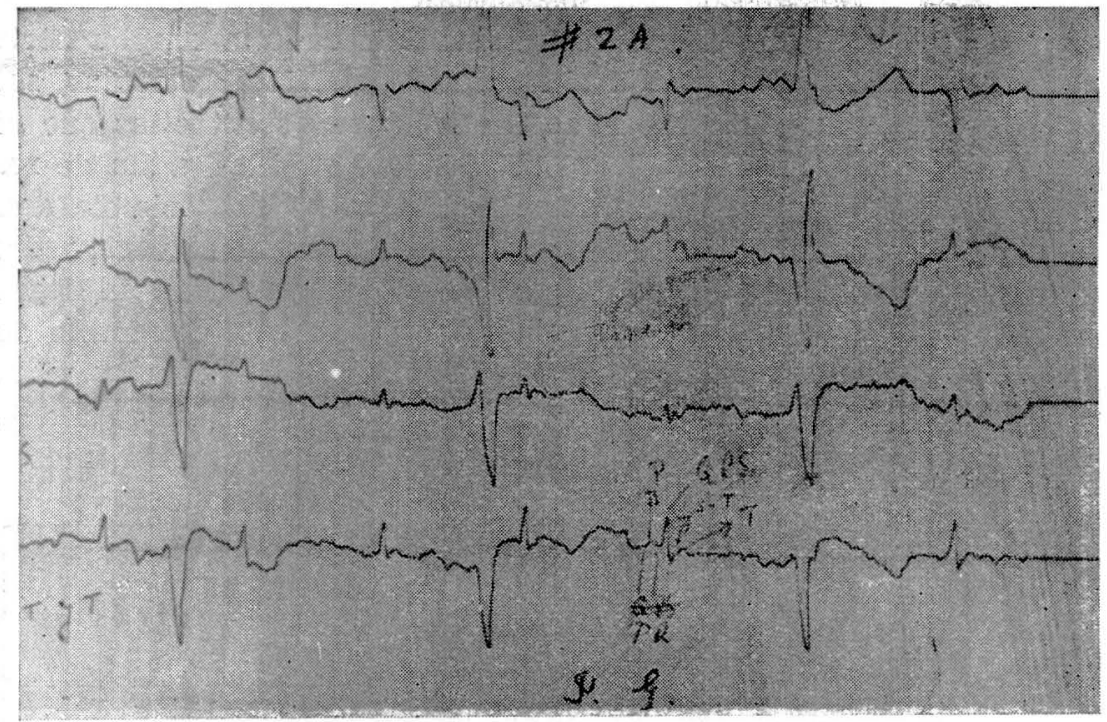

FIGURA NUMERO 2_A

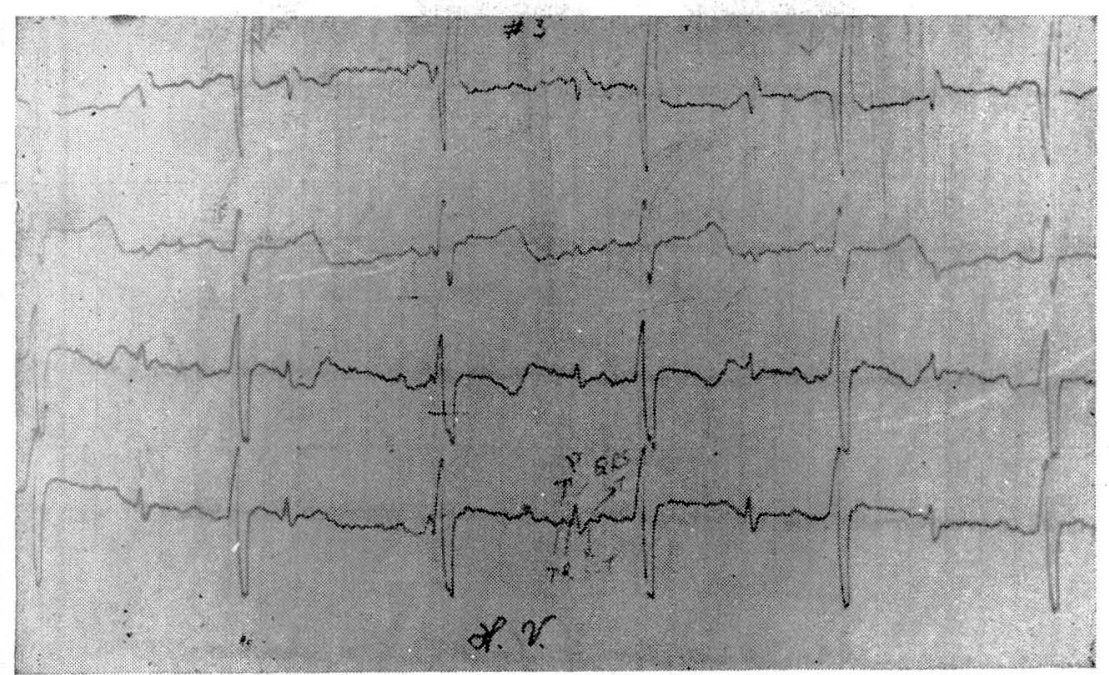

FIGURA NUMERO 3 


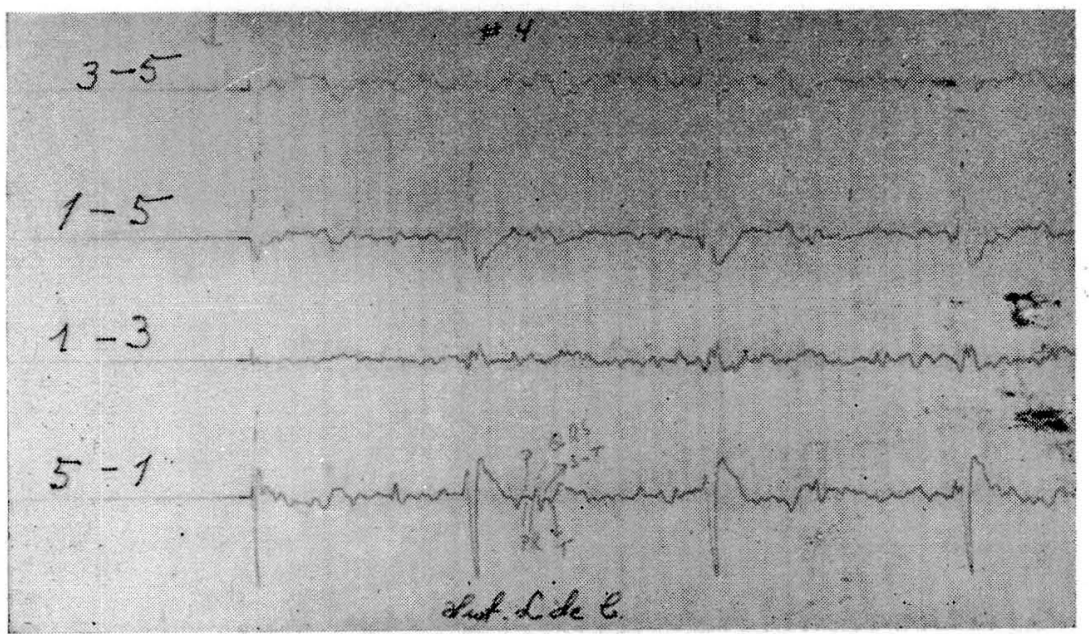

FICURA NUMERO 4

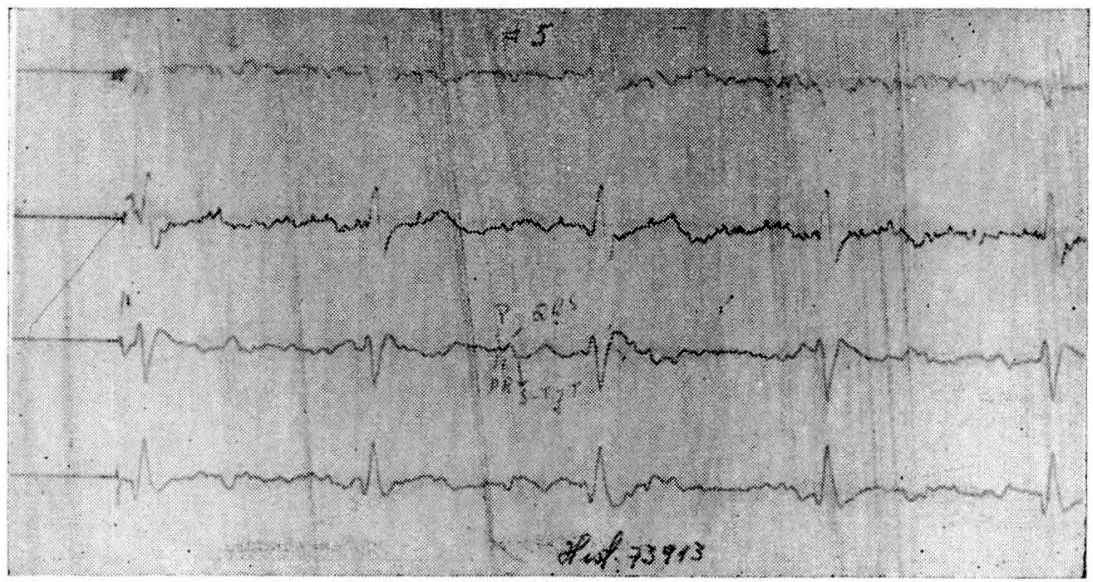

FIGURA NUMERO 5 


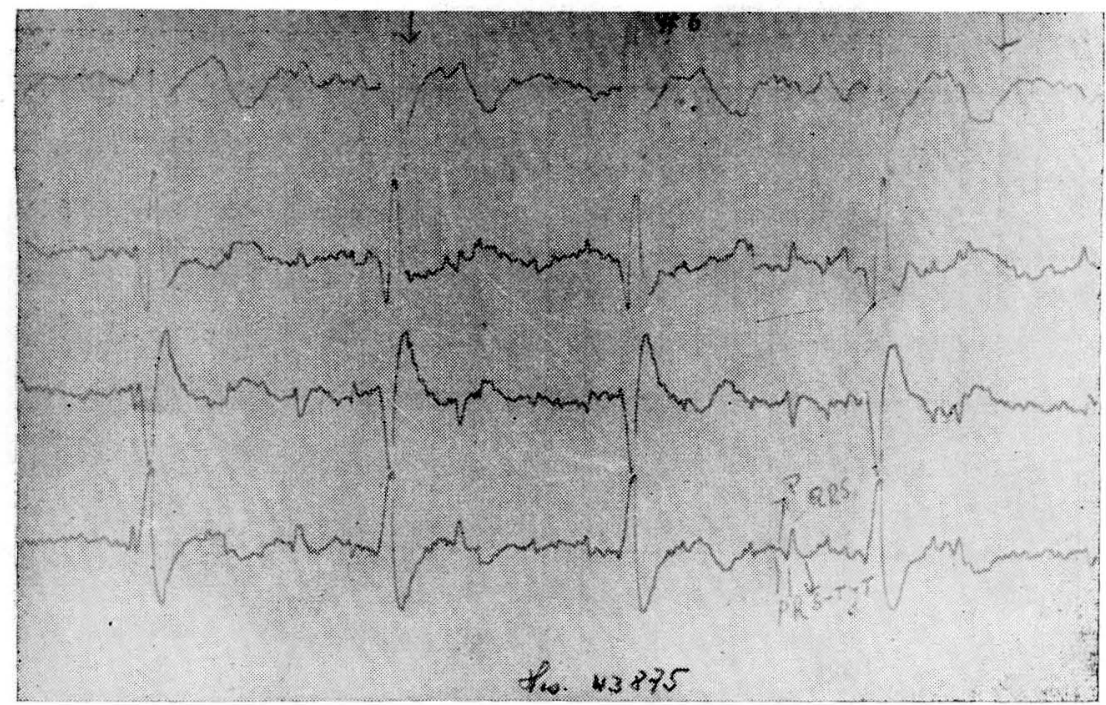

FICURA NUMERO 6

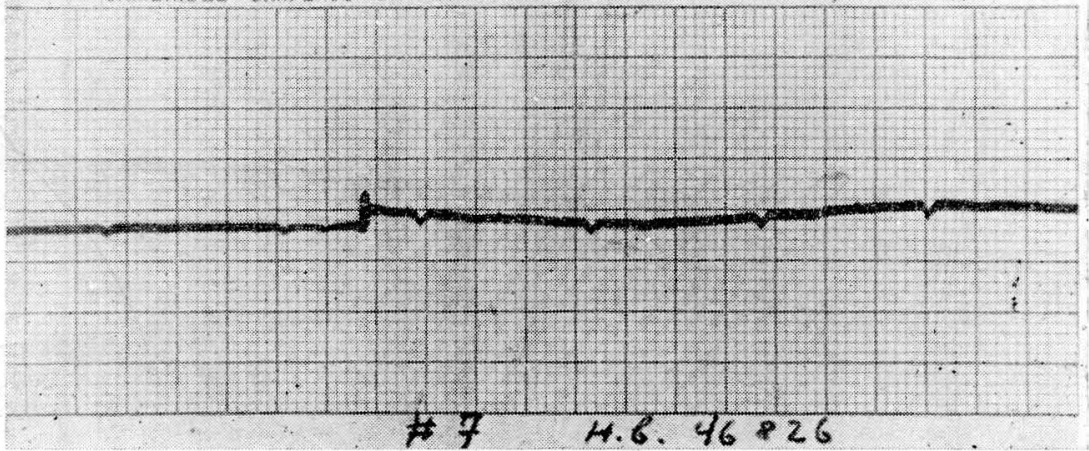

FICURA NUMERO 7 


\section{BIBLIOGRAFIA}

\section{A) Colombiana :}

1. BARRERA J. y FERNANDEZ R.-Algunas constantes en electrocardiogramas normales. Monografía del Curso de Fisiología de la Facultad Nacional de Medicina. Bogotá, 1940.

2. DELGADILLO A. y VILLAMIL C.-Observacicnes y anotaciones de electrocardicgrafía normal. Monografía del Curso de Fisiología de la Facultad Nacional de Medicina de Bogotá, 1940.

3. FORERO NOUGUES LUIS.-El electrocardiograma dermal del lactante. Revista Colombiana de Pediatría y Puericultura, número 2, página 50.

4. GAITAN YANGUAS MARIO.-Ensayo sobre el electrocardiograma en relación con la clínica. Tesis meritoria de la Facultad Nacional de Medicina. 1943, Bogotá.

5. LLINAS JUAN PABLO.-Investigaciones sobre electrocardiografía en Bogotá. Tesis de la Facultad Nacional de Medicina de Bogotá. 1928.

6. MERCHAN R. y MARMOLEJO V.-Apuntes scbre electrocardiografia. Monografía sobre el Curso de Fisiología de la Facultad Nacional de Medicina de Bogotá. 1940 .

7. ORDOÑEZ J. H.-Electrocardiograma normal en Bogotá y su relación con los signos electrocardiográficos de la anoxemia. Revista de la Facultad Nacional de Medicina de Bogotá. Volumen, XI; número, 3. 1942.

8. SANCHEZ ACUÑA JULIO. (+)-Introducción al estudio del electrocardiograma del niño en el edema distrófico. Tesis de la Facultad Nacional de Medicina de Bogotá. 1950.

9. VEJARANO LAVERDE ALBERTO.-Electrocardiografía en recién nacidos. Tesis de la Facultad de Medicina de Bogotá. 1945.

\section{B) Extranjera}

1. GLENDY R. E. and M. M.-Electrocardiography in infants and small children. Suggestions on technic. Am. Heart Jour. 1937. XIV. 66.

2. SCHAUB F.-L'Electrocardiographie. Documenta Geigy, p., 396, 55.

3. SOUTHERN N. E.-Anoxia fetal y su posible relación con cambios en el electrocardiograma fetal pre-natal. Am. J. Obst. Gynec., 11 1957, vol. 73, número 2, página, 233.

4. STEWART J. H.-El electrocardiograma en las enfermedades. Selecciones Médicas de La Habana, III y X-45. Tomo IV, números 19, 20, 21 y 22, página 31 .

5. BRAZIER M., FINESWEZER J. y SCHWABT.-Estudio del electroencefalograma normal. Misma revista anterior, página, 9. 